\title{
||||||||||||||||||||||||||||||||||||||||||||||||||||||||||||||||||||||.
}

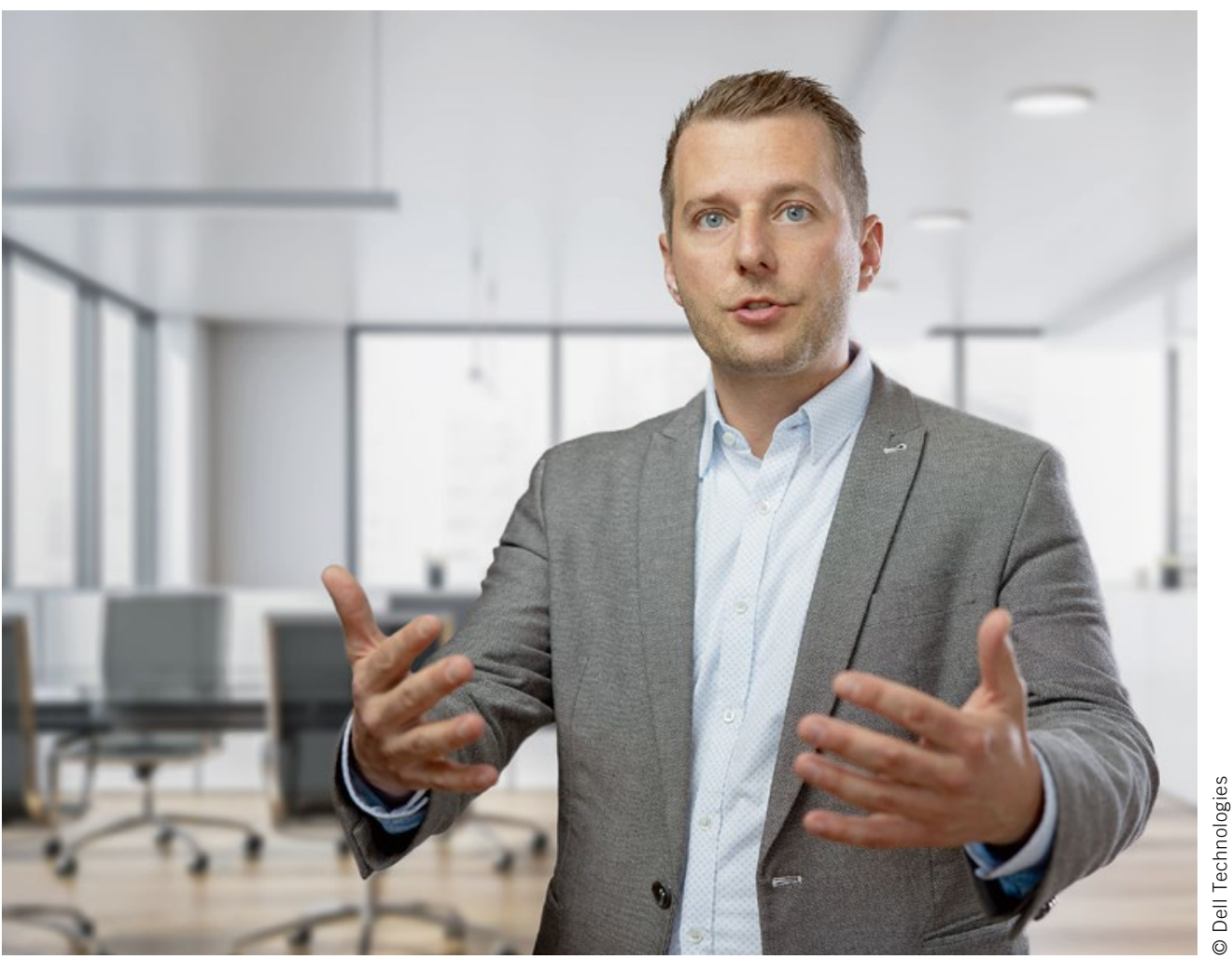

Dr. Florian Baumann

CTO Automotive and Artificial

Intelligence at Dell Technologiess

\section{Data Will Fuel the Mobility of Tomorrow}

How do you get an autonomous vehicle to comply with the rules of the road? You have to make sure it learns, learns, and then learns some more. Highly complex onboard technologies like advanced driver assistance systems, which offer the vehicle a $360^{\circ}$ view of the road and traffic environment with the support of sensors, serve as the basis for this. Lidar, cameras, and radars provide the autonomous vehicle with the sense of sight - but only the intelligent fusion of all data and a corresponding perception algorithm make it possible for the car to independently make decisions.

Design, development, and testing of these driver assistance systems are very complex: The automotive manufacturers have to map many different traffic scenarios. Such scenarios range from road and environmental geometry, to the behavior of drivers and pedestrians in different traffic and weather conditions, right through to interactions between individual sensors or even the failure of entire components. The higher the degree of automation, the more data the automotive industry has to capture using its development vehicles. SAE level 3 typically requires one million travelled $\mathrm{km}$ to be able to simulate enough conditions for algorithm development, testing, validation, and homologation. Level 5 automation - or fully automated driving - calls for about 240 million km. Merely one level-3 project involves the capture of about 19.3 petabytes of raw data for the aforementioned mileage, and we will enter exabyte territory for level 5. Such an enormous transfer of data between the onboard system and the central IT inevitably results in the infrastructure and data system becoming overloaded when conventional approaches are used. The computational complexity simply becomes too immense.

Without real intelligence and a fully integrated development platform, this problem cannot be solved. The platform provides automated processes and workflows that make it possible to evaluate data sets in real-time during the ingestion process from the vehicle to the infrastructure. Then the sensor data including meta-information is preprocessed and compressed with the help of algorithms. This facilitates and safeguards more cost-effective storage solutions. Real-time processing of the data from the vehicle in the cloud and vice versa also requires a low latency at all times.

Autonomous driving is simply not possible without big data, artificial intelligence, and 5G, and what is more: The future of connected mobility strongly relies on an efficient and fully integrated solution for data acquisition, data preparation, and data management. 